Artículo de Investigación

\title{
COVID 19 y embarazo en Chile: Informe preliminar del estudio multicéntrico GESTACOVID
}

\author{
COVID-19 and pregnancy in Chile: preliminary report of the GESTACOVID \\ multicenter study
}

Olivia Hernández B. ${ }^{1}$, Magdalena Honorato S..$^{2,3}$, María Carolina Silva G. ${ }^{3,4}$, Álvaro Sepúlveda-Martínez ${ }^{4}$ Javiera Fuenzalida C. ${ }^{5}$ Fernando Abarzúa C.6,7, Sofía Von Bischhoffshausen P. ${ }^{2}$, Nereida Morales M. ${ }^{8}$, Paz García ${ }^{24}$, María Ignacia Oyarce M. ${ }^{9}$, Gérica Marcano C ${ }^{10}$, Angélica Díaz R. ${ }^{11}$, Rafael Valdés V. ${ }^{12}$, Pamela Vallejos R. ${ }^{13}$, Cristóbal Jacobsen C. ${ }^{14}$, Francisca De Jourdan H. ${ }^{15}$, Rosa Barrios R. ${ }^{16}$, Andrea Vega B. ${ }^{17}$, Trinidad García-Huidobro M. ${ }^{18}$, Francisca Niemann P. ${ }^{19}$, Ulises Guajardo B. ${ }^{20}$, Javier Fuentealba L. ${ }^{21}$, Dominique Agurto G. ${ }^{22}$, Pamela Sanhueza G. ${ }^{23}$, Jorge Carrillo T. 2,3, Juan Pedro Kusanovic P. ${ }^{5,24}$, Grupo GESTACOVID*.

1Servicio Obstetricia y Ginecología, Hospital Clínico Félix Bulnes, Santiago.

¿Unidad de Obstetricia, Unidad de Gestión de la Mujer y el Recién Nacido, Hospital Padre Hurtado, Santiago.

3Departamento de Ginecología y Obstetricia, Facultad de Medicina, Clínica Alemana-Universidad del Desarrollo, Santiago, Chile

4 Unidad de Medicina Materno-Fetal, Departamento de Obstetricia y Ginecología Hospital Clínico, Universidad de Chile, Santiago.

5Departamento de Obstetricia. División de Obstetricia y Ginecología Escuela de Medicina, Pontificia Universidad Católica de Chile, Santiago.

6Servicio Medicina Materno-Fetal, Hospital Regional de Temuco, Temuco.

7Servicio Obstetricia y Ginecología, Clínica Alemana de Temuco, Temuco.

¿Servicio de Obstetricia y Ginecología, Hospital El Pino, Santiago.

9Servicio de Obstetricia y Ginecología, Hospital El Carmen Dr. Luis Valentín Ferrada, Santiago.

10Servicio de Obstetricia y Ginecología, Hospital de Iquique Dr. Ernesto Torres Galdames, Iquique.

${ }^{11}$ Servicio de Obstetricia y Ginecología, Hospital Santiago Oriente Dr. Luis Tisné Broussé, Santiago.

12Servicio de Obstetricia y Ginecología, Clínica Santa María, Santiago.

13Servicio de Obstetricia y Ginecología, Hospital San Juan de Dios de Curicó, Curicó.

${ }^{14}$ Servicio de Obstetricia y Ginecología, Hospital San Pablo de Coquimbo, Coquimbo.

${ }^{15}$ Servicio de Obstetricia y Ginecología, Hospital Regional de Rancagua Libertador Bernardo O’Higgins, Rancagua.

16Servicio de Obstetricia y Ginecología, Hospital Regional de Arica Dr. Juan Noé Crevani, Arica.

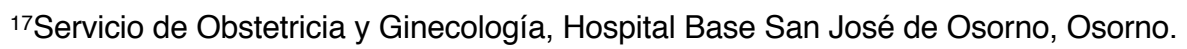

18Servicio de Obstetricia y Ginecología, Hospital Base de Valdivia, Instituto de Obstetricia y Ginecología Universidad Austral, Valdivia.

19 Servicio de Obstetricia y Ginecología, Hospital de Puerto Montt Dr. Eduardo Schütz Schroeder, Puerto Montt. 20Servicio de Obstetricia y Ginecología, Clínica Universidad de los Andes, Departamento de Obstetricia, Ginecología y Biología de la Reproducción, Facultad de Medicina, Universidad de los Andes, Santiago. 
${ }^{21}$ Servicio de Obstetricia y Ginecología, Hospital Regional de Copiapó, Copiapó.

${ }^{22}$ Servicio de Obstetricia y Ginecología, Hospital de Linares, Linares.

${ }^{23}$ Servicio de Obstetricia y Ginecología, Hospital Provincial de Ovalle Dr. Antonio Tirado Lanas, Ovalle.

${ }^{24}$ Servicio de Obstetricia y Ginecología, Centro de Investigación e Innovación en Medicina Materno-Fetal

(CIMAF), Hospital Sótero del Río, Santiago.

Correspondencia: Dr. Jorge Carrillo T.

Email del autor: jcarrillo@alemana.cl jct@vtr.net

\title{
RESUMEN
}

Introducción: En Chile, los efectos maternos y perinatales de la pandemia por SARS-CoV-2 son aún desconocidos. GESTACOVID es un estudio multicéntrico que incluye embarazadas y puérperas hasta el día 42 con COVID-19. El objetivo de este estudio es presentar un informe preliminar, describiendo el impacto de la enfermedad en las embarazadas, factores de riesgo asociados y resultados perinatales.

Métodos: Estudio de cohorte descriptivo que incluye 661 pacientes enroladas entre el 7 de marzo y el 6 de julio de 2020, en 23 centros hospitalarios del país. Se analizaron variables demográficas, comorbilidades, características clínicas y del diagnóstico de COVID-19 y resultado materno y perinatal.

Resultados: Las pacientes hospitalizadas por COVID-19 tuvieron mayor prevalencia de hipertensión arterial crónica [10\% vs $3 \%$; OR=3,1 (1,5-6,79); $p=0,003]$ y de diabetes tipo 1 y $2[7 \%$ vs $2 \%$; OR=3,2 $(1,3-7,7)$; $\mathrm{p}=0,009]$ que las pacientes manejadas ambulatoriamente. Un IMC $>40 \mathrm{~kg} / \mathrm{mt} 2$ se asoció con un riesgo dos veces mayor de requerir manejo hospitalizado [OR=2,4 $(1,2-4,6) ; p=0,009]$. Aproximadamente la mitad de las pacientes (54\%) tuvo un parto por cesárea, y un $8 \%$ de las interrupciones del embarazo fueron por COVID-19. Hasta la fecha de esta publicación, 38\% de las pacientes continuaban embarazadas. Hubo 21 PCR positivas en 316 neonatos $(6,6 \%)$, la mayoría (17/21) en pacientes diagnosticadas por cribado universal.

Conclusiones: Las embarazadas con COVID-19 y comorbilidades como diabetes, hipertensión crónica y obesidad mórbida deben ser manejadas atentamente y deberán ser objeto de mayor investigación. La tasa de transmisión vertical requiere una mayor evaluación para diferenciar el mecanismo y tipo de infección involucrada.

Palabras claves: SARS-CoV-2; pandemia; infecciones virales; embarazo; transmisión vertical; neumonía atípica; coronavirus; síndrome de distress respiratorio.

\begin{abstract}
Introduction: In Chile, effects of the SARS-CoV-2 infection in pregnant women are unknown. GESTACOVID is a multicenter collaborative study including pregnant women and those in the postpartum period (until 42 days) who have had COVID-19. The purpose of this study is to report our preliminary results describing the clinical impact of COVID-19 in pregnant women, the associated risk factors and perinatal results.

Methods: Descriptive cohort study including 661 patients between April 7th and July 6th, 2020, in 23 hospitals. Demographical, comorbidities, clinical and diagnostic characteristics of COVID-19 disease and maternal and perinatal outcomes were analyzed.

Results: Pregnant women with COVID-19 admitted to the hospital were more likely to have chronic hypertension [10\% vs 3\%; OR=3.1 (1.5-6.79); $p=0,003$ ] and diabetes type 1 and 2 [7\% vs $2 \%$; OR=3.2 (1.3-7.7); $p=0.009]$ than those with outpatient management. A body mass index of $>40 \mathrm{~kg} / \mathrm{mt} 2$ was associated with two-fold higher risk of hospitalization [OR=2.4 (1.2-4.6); $\mathrm{p}=0.009$ ]. Almost half of patients $(54 \%)$ were delivered by cesarean section, and $8 \%$ of the medically indicated deliveries were due to COVID-19. So far, $38 \%$ of the patients are still
\end{abstract}


pregnant. Among 316 newborns, there were 21 positive PCR tests (6.6\%), mostly from asymptomatic mothers undergoing universal screening.

Conclusions: Pregnant women with COVID-19 and comorbidities such as diabetes, chronic hypertension and morbid obesity need a close follow up and should be a matter for further research. Vertical transmission of COVID-19 should be thoroughly studied to define the mechanisms and type of infection involved.

Keywords: SARS-CoV-2; pandemic; viral infections; pregnancy; vertical transmission; atypical neumonia; coronavirus; respiratory distress syndrome.

\section{INTRODUCCIÓN}

La nueva enfermedad por Coronavirus (COVID19) es la tercera propagación documentada de un coronavirus animal en humanos que ha resultado en una pandemia1. Las anteriores epidemias causadas por $\beta$-coronavirus zoonóticos, SARS-CoV y MERSCoV, que comparten similitudes con SARS-CoV-2 en la estructura genética de los $\beta$-coronavirus, reportaron tasas de letalidad materna de $10 \%$ y $37 \%$ respectivamente, en las que la morbimortalidad materna y perinatal no estuvo exenta2-3.

Los cambios adaptativos fisiológicos, mecánicos e inmunológicos en el embarazo sitúan a las gestantes en condición de susceptibilidad a contraer infecciones respiratorias, las que pueden progresar a una insuficiencia respiratoria aguda grave. La evidencia disponible sugiere que la interrupción de la gestación reduce de manera inmediata un $28 \%$ de los requerimientos de oxígeno, lo que facilitaría la reanimación y estabilización materna en el contexto de una falla respiratoria4-8.

En la actual pandemia, existe creciente evidencia en relación con el efecto de COVID-19 en el embarazo. Una revisión sistemática reciente 9 reporta una tasa de embarazadas sintomáticas del 67\%, siendo fiebre, tos y malestar general los síntomas más frecuentes en un $61 \%, 38$ y $30 \%$ respectivamente y sólo un $12 \%$ de las embarazadas se presentó con disnea. La tasa de admisión a Unidades de Pacientes Críticos (UPC) se comunica entre un 3-4\%9, y entre 1,3 a 1,4\% se presentaron con patología crítica ${ }^{9,10}$.

La evidencia actual es limitada respecto a la tasa de transmisión vertical. Las cifras de recién nacidos con PCR positiva oscilan entre 0 y $5 \%$ 9; sin embargo, los reportes son heterogéneos en cuanto al momento de la toma del examen, por lo que no se puede descartar de manera definitiva una transmisión horizontal 9-11.
En nuestro país aún no existen datos publicados de pacientes embarazadas con COVID-19, lo que nos impide conocer el tipo de complicaciones y la frecuencia con que pueden afectar a nuestras pacientes. Por lo anterior, surge la iniciativa de crear la red colaborativa GESTACOVID, un estudio multicéntrico que agrupa a diversas maternidades tanto de instituciones públicas y privadas, que podrá caracterizar el comportamiento de este virus en las embarazadas de nuestro país.

El objetivo del presente estudio es dar a conocer un informe preliminar de GESTACOVID, informando la proporción de infectadas que requieren manejo en UPC, factores de riesgo asociados y resultados perinatales a la fecha.

\section{MATERIAL Y MÉTODOS \\ Diseño del estudio}

El estudio GESTACOVID es una cohorte multicéntrica descriptiva que incluye embarazadas y puérperas hasta el día 42 con diagnóstico de infección por SARS-CoV-2, que cuenta con la aprobación de los Comités Éticos Científicos (CEC) locales o del Director(a) de la institución y programado para ser realizado entre Marzo de 2020 y Marzo de 2021. La presente comunicación busca dar a conocer cifras preliminares del estudio, incluyéndose datos de 23 instituciones de nuestra red de hospitales y clínicas de Chile, con información disponible de los primeros meses del estudio. Dado que la información para este estudio acotado fue recolectada sin acceso directo a las pacientes y eliminando la información sensible y anonimizada al entregarla a los investigadores para un análisis de data agregada, no se requirió consentimiento de las pacientes.

Las variables incluidas fueron: características demográficas al momento del diagnóstico de la infección [edad, índice de masa corporal (IMC)], 
patología crónica [hipertensión arterial (HTA) crónica, diabetes mellitus (DM)], características clínicas de la enfermedad COVID-19 [indicación del hisopado para PCR (tamizaje universal al hospitalizar para estudio, para interrupción del embarazo o en trabajo de parto; contacto de paciente sospechoso; pacientes sintomáticas), síntomas al diagnóstico, necesidad de hospitalización, ingreso a Unidad de Paciente Crítico (UPC)], características perinatales y neonatales incluyendo vía del parto, edad gestacional al parto, asociación con infección por SARS-CoV-2, prematuridad, peso del neonato, diagnóstico de pequeño para la edad gestacional (PEG), tamizaje y resultado de RT-PCR en neonatos, morbilidad materna grave y presencia de muerte materna o neonatal. Todas las variables fueron almacenadas en una planilla Excel (Microsoft 365, Microsoft, Redmon, WA, USA) local anonimizada y protegida para su posterior fusión previo al análisis.

Selección de pacientes y definiciones del estudio.

Para el presente estudio se incluyen embarazadas y puérperas en seguimiento hasta el día 42, con el diagnóstico de infección por SARS-CoV-2, realizado mediante Reverse Transcription-Polymerase Chain Reaction (RT-PCR)12-13 y/o serología que indique enfermedad activa [Inmunoensayo de flujo lateral para $\lg$ e e lgG - Lateral Flow Immuno Assay (LFIA) en inglés], o imágenes de tórax características de neumonía por virus SARS-CoV-2 en pacientes sintomáticas, según la clasificación del Consenso de Expertos para informar hallazgos relacionados con COVID-19, de la Sociedad Radiológica de Norteamérica $^{14}$. Se excluyeron pacientes que tuvieran edad gestacional dudosa o que no tuvieran pruebas de confirmación por serología, PCR o imagenología concordante.

Se considera parto prematuro aquel que ocurre antes de las 37 semanas, y recién nacido pequeño para la edad gestacional (PEG) a todo aquel con un peso de nacimiento bajo el percentil 10 de la curva de peso nacional ${ }^{15-17}$; no se dispuso de información sobre la curva de crecimiento o el Doppler fetal antes del nacimiento.

\section{Análisis estadístico}

Las variables categóricas son expresadas como proporciones. La distribución de las variables continuas es analizada con la prueba Shapiro-Wilk, siendo expresadas como media \pm desviación estándar o mediana (rango intercuartil) para aquellas con distribución normal o no paramétrica, respectivamente. Para la comparación de proporciones se realiza la prueba chi cuadrado o el test exacto de Fisher, según correspondiera. Para dicotomizar la edad materna y el IMC, se obtiene el mejor valor de corte por medio del análisis de curva ROC. La determinación de riesgo se realiza por medio de una regresión logística simple, expresándose como Odds Ratio (IC 95\%). Para todos los análisis se considera como significativo un valor $\mathrm{p}<0.05$. Para los análisis estadísticos se utilizó el software Stata 16.1 (Statacorp, College Station Texas, USA).

\section{RESULTADOS}

Este estudio incluyó 661 pacientes embarazadas y puérperas con el diagnóstico de COVID-19, enroladas desde el 7 de marzo al 6 de julio de 2020 en 23 centros de nuestro país (Figura 1), distribuidos entre la zona norte (9\%), centro $(87 \%)$ y sur $(4 \%)$ (Tabla 1).

Las características demográficas, clínicas y del diagnóstico de COVID-19 de las pacientes incluidas en este estudio se muestran en la Tabla 2. Aproximadamente el $86 \%$ de las embarazadas presentó sobrepeso u obesidad al momento del diagnóstico, un $5 \%$ eran hipertensas crónicas y un $3 \%$ diabéticas pregestacionales. La mayoría de las pacientes fueron diagnosticadas en el tercer trimestre y sólo un 6\% durante el puerperio (Tabla 2).

Un $51 \%$ de las embarazadas fue estudiada por sintomatología sugerente de COVID-19, el 10\% tuvo el examen por ser contacto directo de un paciente COVID-19 y un 39\% como parte del cribado universal al ingreso para estudio en Alto Riesgo Obstétrico, para interrupción programada del embarazo o en trabajo de parto (Tabla 3). Los síntomas más frecuentes fueron tos $(35 \%)$, cefalea $(31 \%)$, mialgias $(30 \%)$ y fiebre (29\%), siendo significativamente más prevalentes en las pacientes que requirieron hospitalización en comparación con las que tuvieron un manejo ambulatorio. La disnea se presentó en $21 \%$ de las pacientes, siendo 22 veces más frecuente en pacientes hospitalizadas que en aquellas con manejo 
ambulatorio (71\% vs $10 \%$; (OR= 22,4 (IC 13,9-36,4) (Tabla 3, Figura 2).

En esta cohorte, 129 de 659 pacientes (19\%) con infección por SARS-CoV-2 debieron ser hospitalizadas para su manejo, y 47 de las 129 (39\%) debieron ser admitidas a UPC (Tabla 2). Las pacientes hospitalizadas por COVID-19 tuvieron una mayor prevalencia de HTA crónica [10\% vs $3 \%$; $\mathrm{OR}=3,1(1,5-6,8) ; \mathrm{p}=0,003]$ y de DM tipo 1 y tipo $2[7 \%$ vs $2 \%$; OR=3,2 $(1,3-7,7) ; p=0,009$ ] que las pacientes manejadas ambulatoriamente. No hubo diferencias significativas en la mediana del IMC entre pacientes que requirieron manejo hospitalizadas por COVID en comparación con las manejadas ambulatoriamente [mediana $=30,5(27,2-37,0)$ vs. $30,3(26,7-34,4)$; $\mathrm{p}=0,2]$. Sin embargo, un IMC $>40 \mathrm{~kg} / \mathrm{mt} 2$ se asocia con un riesgo dos veces mayor de requerir manejo hospitalizado [OR=2,4 $(1,2-4,6) ; p=0.009]$. No hubo diferencias en la mediana del IMC entre las pacientes hospitalizadas en sala básica comparadas con las ingresadas a la UPC.

El antecedente de DM tipo 1 y tipo 2 se asoció con un riesgo tres veces mayor de necesitar hospitalización en una UPC, comparado con las embarazadas sin DM pregestacional [OR=3,5 $(0,70-$ 22,6); $p=0,07]$. De hecho, las pacientes que se hospitalizaron eran significativamente mayores que aquellas que no se hospitalizan [mediana: 30,7 años $(27,6-34,2)$ vs. 29,6 años $(24,8-34,4) ; p=0,027$, respectivamente]. Al definir un valor de corte con curva ROC, una edad materna $\geq 38$ años se asocia con un riesgo 3 veces mayor de necesidad de hospitalización en una UPC [OR=3,4 $(1,17-9,98)$; $p=0,025]$ que las embarazadas de $<38$ años.

El resultado perinatal puede verse desplegado en la Tabla 4. La mediana de la edad gestacional al parto y el peso del recién nacido fue de 38,6 semanas y $3270 \mathrm{grs}$, respectivamente, con una tasa de neonatos PEG de 9\%, similar a la población general de embarazadas del país. Aproximadamente la mitad de las pacientes tuvo su parto por cesárea (54\%), y $8 \%$ de las interrupciones tuvieron como indicación una complicación relacionada con COVID-19 o que a juicio del equipo tratante, la interrupción facilitara el manejo de su patología; no se cuenta con el detalle para este reporte las que para este reporte no fueron detalladas. Hubo 68 partos (16\%) antes de las 37 semanas, y la tasa de muerte perinatal fue de $1 \%$ (6 casos). Hasta la fecha del envío de esta publicación, 254 de las 661 pacientes $(38 \%)$ continuaban embarazadas.

De los 389 recién nacidos vivos, 316 tuvieron un resultado del examen de PCR obtenido luego del parto. De esas, $93 \%$ (293/316) resultaron negativas, $6 \%(21 / 316)$ positivas y $1 \%(2 / 316)$ no concluyente. De los 21 recién nacidos con una PCR positiva para SARS-CoV-2, 17 provenían de madres identificadas por cribado universal (81\%), tres en pacientes sintomáticas (14\%) y un caso $(5 \%)$ en que la madre era contacto estrecho de un paciente COVID-19 (Tabla 5). En 10 casos la prueba se realizó dentro de 12 horas de vida, en 2 casos entre 12 y 24 horas post parto, en 5 casos se realizó más de 48 horas post parto por síntomas sugerentes de COVID-19 de la paciente y en un caso el test se repitió luego de un primer test negativo a las 12-24 hrs del parto, porque la madre estaba sintomática de COVID-19.

\section{DISCUSIÓN}

\section{Hallazgos principales del estudio}

La presente comunicación es, según nuestra información, la primera iniciativa de un registro multicéntrico nacional que reporta las características clínicas maternas y perinatales de 661 embarazadas o puérperas con diagnóstico de COVID-19.

Hasta hace pocos meses no se tenía antecedentes de la infección provocada por el nuevo coronavirus SASRS-CoV-2 en humanos; por lo tanto, se dispone de poca información en relación con las características de esta enfermedad y su curso natural. Los reportes en pacientes embarazadas y puérperas son aún escasos, y las complicaciones que pueden traer en la gestante y su hijo son todavía poco conocidas. En brotes epidémicos, principalmente documentados en influenza, las embarazadas se comportan como un grupo de alto riesgo para hospitalización, morbilidad grave, e incluso muerte ${ }^{18}$ 20. Por lo tanto, es de suma importancia conocer el impacto que tiene y tendrá la enfermedad COVID-19 en el embarazo.

El estándar de oro para el diagnóstico de infección por SARS-CoV-2 es la RT-PCR ${ }^{12-13,21}$. En nuestra serie, el $99 \%$ de las pacientes fueron confirmadas a través de PCR en hisopado nasofaríngeo. Este reporte preliminar cuenta con $51 \%$ de las pacientes 
detectadas por ser sintomáticas para COVID-19, y $10 \%$ por ser contacto estrecho de un caso confirmado; solo un $39 \%$ de nuestra serie fue por cribado universal al hospitalizarse por patología obstétrica, en trabajo de parto, o antes de una interrupción electiva por inducción o cesárea.

El promedio de edad de las pacientes es similar a lo reportado en otras series ${ }^{22}$. En este reporte, una edad materna $\geq 38$ años otorga un riesgo de tres veces mayor de hospitalización en una UPC, información que puede ser relevante para realizar una vigilancia más estrecha en este grupo de embarazadas. La obesidad y la presencia de comorbilidades han sido factores descritos en otros reportes ${ }^{22,23}$, siendo la primera una condición señalada como constante y asociada a mayor riesgo de enfermar y de presentar una evolución más grave de la enfermedad, riesgo que se aplica también a la población de embarazadas ${ }^{24-26}$. En nuestro estudio, la mediana del IMC fue de 30,4, cifra concordante con la tendencia al exceso de peso de nuestra población ${ }^{27}$. No se apreciaron diferencias significativas entre las pacientes con COVID-19 que requirieron hospitalización y las manejadas ambulatoriamente. Sin embargo, un IMC >40 (obesidad mórbida) otorga un riesgo dos veces mayor de hospitalización.

La prevalencia de DM tipo 1 y 2 e HTA crónica (3\% y $5 \%$, respectivamente) es relativamente baja en la serie global; sin embargo, en el grupo de pacientes hospitalizadas, el antecedente de DM tipo 1 y 2 incrementa en tres veces el riesgo de ingreso a UPC, dato clínico a considerar para reforzar la necesidad de cuidado dirigido y confinamiento físico para reducir el riesgo de contagio al máximo; y si requieren hospitalización, para la vigilancia estricta de la evolución de estas pacientes en caso de ingresar inicialmente en una sala básica.

En relación con el cuadro clínico, se advierten diferencias significativas entre la sintomatología de las embarazadas manejadas en forma ambulatoria y las hospitalizadas. Un elemento diferenciador importante es la disnea, presente en el $71 \%$ de las hospitalizadas y sólo en el $10 \%$ de las que se manejaron en forma ambulatoria. La fiebre estuvo presente en un $29 \%$ de las pacientes, pero solamente en $20 \%$ de las ambulatorias y $68 \%$ en las pacientes con manejo intrahospitalario. La tos está presente en $78 \%$ de las hospitalizadas mientras que sólo en el $25 \%$ de las ambulatorias. Esto coincide con la literatura que considera cuadros clínicos de mayor severidad según el tipo de síntomas que presenta la paciente ${ }^{5,23}$.

Nuestra cohorte registra 123 pacientes que se hospitalizaron. Cuarenta y ocho pacientes presentaron morbilidad materna grave con ingreso a UPC en algún momento de su evolución, lo que representa un 39\% de las hospitalizadas. En esta serie no hay muertes maternas por COVID-19. La letalidad ha sido baja en la gran mayoría de los estudios de embarazadas, fluctuando entre 0 y $1 \%$, al igual que el requerimiento de oxigenación por membrana extracorpórea (ECMO)22,23,28. En el estudio UKOSS 21 del Reino Unido, que incluyó 427 embarazadas sintomáticas, el $9 \%$ requirió cuidados críticos y $<1 \%$ recibió $\mathrm{ECMO}$; hubo 5 muertes maternas, lo que representa una tasa de letalidad de 1,2\% (IC 95\% 0,4-2,7) en gestantes con COVID-19. Esto es la mitad de las tasas de letalidad de esta pandemia reportadas para la población general, pudiendo llegar hasta un $49 \%$ en pacientes críticos ${ }^{29}$. No obstante, con más caudal de información es esperable que estas cifras aumenten, ya que una publicación reciente de Takemoto et al. describe 124 muertes en el embarazo o el postparto en Brasil (tasa reportada de $12 \%$ ), lo que sobrepasa el total de muertes maternas por COVID-19 en el resto del mundo 30 .

La tasa de parto prematuro reportada varía entre 15 y $27 \%$ en diversas series, dependiendo del porcentaje de pacientes sintomáticas severas que se incluyan 22,23,28,7,31. En esta cohorte, casi el 18\% de las pacientes presentó un parto prematuro, y en un tercio de los casos, la indicación fue por enfermedad materna grave por COVID-19. El 54\% de los partos fueron por cesárea, coincidiendo con literatura más reciente 32,33 , a diferencia de los primeros reportes de China, en que se describió una alta tasa de prematuridad (21\%- $47 \%$, la mayoría partos prematuros indicados), con una tasa de cesárea de hasta $93 \%$, siendo $61 \%$ de ellas debido a la preocupación por los efectos de COVID-19.34

Hasta el momento, los estudios no han evidenciado de manera clara la presencia del virus en fluidos genitales ni líquido amniótico, por lo que, respecto a la transmisión vertical, la información se 
limita aún a pocos reportes que sugieren una factibilidad teórica. ${ }^{35}$ Cabe señalar que no hay criterios uniformes en los diferentes estudios sobre el momento de toma de la muestra nasofaríngea para la PCR, por lo que ha sido difícil diferenciar entre transmisión vertical de la infección de una infección adquirida en el postparto, donde el vector puede ser su madre o el mismo equipo de salud ${ }^{25,36}$. La tasa de recién nacidos con PCR positiva para COVID-19 fluctúa entre 1 y $5 \%{ }^{9-11}$, siendo en nuestra serie de un $6 \%$ (21 casos). Llamativo resulta que sólo en tres de los casos la madre estaba sintomática, en el $86 \%$ restante el estudio materno fue por tamizaje universal o antecedente de contacto estrecho. La primera experiencia publicada de tamizaje con PCR a todas las pacientes que ingresaron en trabajo de parto $(n=215)$ se publicó en marzo de 2020 en Nueva York. En 33 de ellas la prueba fue positiva para SARS-CoV2,4 de ellas con fiebre al ingreso y 29 asintomáticas, lo que representa que un $87 \%$ de las pacientes positivas eran asintomáticas y detectadas por tamizaje. ${ }^{25}$ Esto reafirma la importancia de incorporar el cribado universal materno como estrategia para reducir el riesgo de contagio al personal de salud y al recién nacido en los días posteriores al parto, lo cual ha sido recomendado por diferentes sociedades científicas en nuestro país. ${ }^{37}$

\section{Fortalezas y debilidades}

Entre las fortalezas del presente estudio está su carácter representativo de diferentes centros hospitalarios del país, con diverso nivel de complejidad, condición socioeconómica de la población, comorbilidades y tipo de población migrante, entre otros. Esta información se hace muy necesaria al momento de planificar medidas de prevención, de vigilancia y de intervenciones más eficaces. Las pacientes hospitalizadas representan el grupo de mayor riesgo y esta serie es probablemente representativa de ese subgrupo de embarazadas. También debemos considerar un logro el establecimiento de una red de colaboración directa de las maternidades para producción científica, que tenga impacto en las políticas de salud de nuestra población.

El estudio tiene limitaciones; pensamos que hay un subregistro de gestantes afectadas por COVID-19, ya que la misma pandemia ha hecho que menos embarazadas se acerquen a la atención primaria en el primer y segundo trimestre del embarazo. Asimismo, sin una estrategia de tamizaje universal a todas las embarazadas, muchas pacientes asintomáticas no son reconocidas. La pesquisa de infección con hisopado nasofaríngeo al término no es una conducta homogénea en los centros de salud. Tampoco este estudio solicitó la evaluación del crecimiento ecográfico ni el Doppler fetal, por lo que nuestra cifra de neonatos PEG no se pudo correlacionar con el estatus fetal antenatal. Este es un registro basado en la captación de embarazadas que concurren a atención a los centros de salud por consulta espontánea o para su parto y no basado en población, lo que nos hace considerar estos resultados con prudencia.

Este reporte ejecutivo se ha producido cuando la pandemia aún está en curso, y la limitación asociada a esto debe ser tomada en cuenta. Aún no han tenido su parto 254 pacientes (38\%) de la serie, siendo esa información potencialmente relevante. Por lo mismo, la información recogida de los centros ha sido solamente lo esencial; falta disponer de más información de las pacientes graves y críticas, y de la evolución de los recién nacidos, especialmente los que tuvieron una PCR positiva. Lo mismo podemos decir de la información sobre estado socioeconómico y los efectos sociales en la población migrante, de lo que no tenemos información para este reporte.

\section{CONCLUSIÓN}

En conclusión, este estudio aporta valiosa información que permite presentar preliminarmente la evolución de la pandemia por SARS-CoV-2 en la población de embarazadas del país. Sus resultados requieren ser estudiados con entusiasmo y a la vez con prudencia: se pueden planear estrategias de pesquisa temprana para reducir la probable infección neonatal. Asimismo, los grupos de embarazadas con edad materna mayor de 38 años, las pacientes diabéticas, hipertensas crónicas y obesas mórbidas deben ser manejadas atentamente y deben ser objeto de mayor investigación. El estudio GESTACOVID contempla aumentar el número de pacientes enroladas e incorporar y evaluar las variables y 
poblaciones previamente mencionadas en su proyecto final.

\section{REFERENCIAS}

1. WHO: Coronavirus disease (COVID-2019) situation report 51 https://www.who.int/docs/defaultsource/coronaviruse/situation-reports/20200311sitrep-51-covid-19.pdf?sfvrsn=1ba62e57_10

2. Wong SF, Chow KM, Leung TN, et al. Pregnancy and perinatal outcomes of women with severe acute respiratory syndrome. Am J Obstet Gynecol. 2004;191(1):292-297.

3. Alfaraj SH, Al-Tawfiq JA, Memish ZA. Middle East Respiratory Syndrome Coronavirus (MERS-CoV) infection during pregnancy: Report of two cases \& review of the literature. J Microbiol Immunol Infect. 2019;52(3):501-503.

4. Mehta N, Chen K, Hardy E, Powrie R. Respiratory disease in pregnancy. Best Pract Res Clin Obstet Gynaecol. 2015;29(5):598-611.

5. Graves CR. Pneumonia in pregnancy. Clin Obstet Gynecol. 2010;53(2):329-336.

6. Lapinsky SE. Acute respiratory failure in pregnancy. Obstet Med. 2015;8(3):126-132.

7. Tomlinson MW, Caruthers TJ, Whitty JE, Gonik B. Does delivery improve maternal condition in the respiratory-compromised gravida? Obstet Gynecol. 1998;91(1):108-111.

8. Daily $\mathrm{WH}$, Katz AR, Tonnesen A, Allen SJ. Beneficial effect of delivery in a patient with adult respiratory distress syndrome. Anesthesiology. 1990;72(2):383-386.

9. Huntley BJF, Huntley ES, Di Mascio D, Chen T, Berghella V, Chauhan SP. Rates of Maternal and Perinatal Mortality and Vertical Transmission in Pregnancies Complicated by Severe Acute Respiratory Syndrome Coronavirus 2 (SARS-CoV-2) Infection: A Systematic Review. Obstet Gynecol. 2020.

10. Smith V, Seo D, Warty $R$, et al. Maternal and neonatal outcomes associated with COVID-19 infection: A systematic review. PLoS One. 2020;15(6):e0234187.

11. Duran P, Berman S, Niermeyer S, et al. COVID19 and newborn health: systematic review. Rev Panam Salud Publica. 2020;44:e54.

12. Diao B., Wen K., Chen J., Liu Y., et al. Diagnosis of Acute Respiratory Syndrome Coronavirus 2 Infection by Detection of Nucleocapsid Protein. www.medrxiv.org preprint doi: https://doi.org/10.1101/2020.03.07.20032524

13. Yang $Y$, Yang $M$ et al. Evaluating the accuracy of different respiratory specimens in the laboratory diagnosis and monitoring the viral shedding of 2019-nCoV infections. medRxiv 2020.02.11.20021493; doi: https://doi.org/10.1101/2020.02.11.20021493

14. Simpson S, Kay F, Abbara S, Bhalla S, Chung JH, Chung M, Henry T, Kanne J, Kligerman S, Ko J, Litt H. Radiological Society of North America Expert Consensus Statement on Reporting Chest CT Findings Related to COVID-19. Endorsed by the Society of Thoracic Radiology, the American College of Radiology, and RSNA. HYPERLINK "C:IIUsersIlchepolIDropboxIIResearchIIGESTACOVIDIIPaper sochog v2llJ"J Thorac Imaging 2020;35(4):219-227.

15. Alarcón J, Alarcón Y, Hering E, Buccioni R. Curvas antropométricas de RN chilenos. Rev Chil Pediatr 2008; 79 (4): 364-372.

16. Pittaluga PE, Díaz AV, Mena $P$ et al. Curva de crecimiento intrauterino para prematuros entre 23 a 36 semanas de edad gestacional. Rev Chil Pediatr 2002; 73 (2): 135-41

17. Milad M, Novoa JM, Fabre J, Samamé $M$, Aspillaga C. Recomendación sobre Curvas de Crecimiento Intrauterino. Rev Chil Pediatr 2010; 81 (3): 264-274

18. Schwartz DA, Graham AL. Potential maternal and infant outcomes from Coronavirus 2019-nCoV (SARS-CoV-2) infecting pregnant women: Lessons from SARS, MERS, and other human coronavirus infections. Viruses. 2020;12:194.

19. Baback Gharizadeh, Junqiu Yue, et al. Navigating the Pandemic Response Life Cycle: Molecular Diagnostics and Immunoassays in the Context of COVID-19 Management. IEEE Rev Biomed Eng. 2020 Apr 29.

20. Abarzúa F. COVID-19 y Embarazo. Disponible en: URL: https://sochog.cl/archivos/12821.

21. Yang $Y$, Yang $M$ et al. Evaluating the accuracy of different respiratory specimens in the laboratory diagnosis and monitoring the viral shedding of 2019-nCoV infections. medRxiv 2020.02.11.20021493; doi: https://doi.org/10.1101/2020.02.11.20021493

22. Khoury $R$, Bernstein $P$, Debolt $C$, et al. Characteristics and Outcomes of 241 Births to Women With Severe Acute Respiratory Syndrome Coronavirus 2 (SARS-CoV-2) Infection 
at Five New York city Medical Centers. Obstet Gynecol 2020;136(2):273-282.

23. Knight $M$, Bunch $K$, Vousden $N$, et al. Characteristics and outcomes of pregnant women admitted to hospital with confirmed SARS-CoV-2 infection in UK: national population based cohort study. BMJ. 2020;369:m2107.

24. Ellington, S. Strid P, Tong V, Woodworth K et cols. Characteristics of women of reproductive age with laboratory-confirmed SARS-CoV-2 infection by pregnancy status - United States, January 22-june 7, 2020. MMWR Morb Mortal Wkly Rep 2020;69(25): 769-775.

25. Sutton D, Fuchs M, D'Alton M, Goffman D. Universal screening for SARS-CoV-2 in women admited for delivery. $N$ Engl $J$ Med. 2020;382(22):2163-2164.

26. Juan J, Gil MM, Rong Z, Zhang Y, Yang H, Poon LC. Effect of coronavirus disease 2019 (COVID19) on maternal, perinatal and neonatal outcome: systematic review. Ultrasound Obstet Gynecol. 2020;56(1):15-27.

27. Araya M, Padilla O, Garmendia ML, Atalah E, Uauy R. Obesidad en mujeres chilenas en edad fértil. Rev Med Chile 2014; 142 (11):1440-1448.

28. Kayem G, Lecarpentier C, Deruelle P, et al. A snapshot of the Covid-19 pandemic among pregnant women in France. J Gynecol Obstet Hum Reprod 2020;49(7):101826..

29. Zunyou Wu, Jennifer M. McGoogan. Characteristics of and Important Lessons From the Coronavirus Disease 2019 (COVID-19) Outbreak in China. Summary of a Report of 72314 Cases From the Chinese Center for Disease Control and Prevention. JAMA. 2020;323(13):1239-1242.
30. Takemoto M, O Menezes M, Andreucci C, et al. The tragedy of COVID-19 in Brazil: 124 maternal deaths and counting. Int J Gynaecol Obstet 2020. doi: 10.1002/ijgo.13300

31. Liu Y, Chen H, Tang K, Guo Y. Clinical manifestations and outcomes of SARS-CoV-2 infection during pregnancy. J Infect. 2020 Mar 4. doi: 10.1016/j.jinf.2020.02.028.

32. Mullins E. et al. Coronavirus in pregnancy and delivery: rapid review. Ultrasound Obstet Gynecol 2020; 55: 586-592.

33. Liu H, Liu F, Li J et al. Clinical and CT imaging features of the COVID-19 pneumonia: focus on pregnant women and children. J Infect 2020;80(5):e7-e13.

34. Guan W, Ni Z, Hu Y, et al. Clinical Characteristics of Coronavirus Disease 2019 in China. Mar 24 2020. N Engl J Med 2020;382(18):1708-1720.

35. RCOG. Coronavirus (COVID-19) infection in pregnancy. Information for healthcare professionals. 4 June 2020. Disponible en: URL: https://www.rcog.org.uk/globalassets/documents/ guidelines/2020-06-04-coronavirus-covid-19infection-in-pregnancy.pdf

36. Schwartz D. An analysis of 38 pregnant women with Covid-19, their newborn infants and maternal fetal transmission of SARS-CoV-2: maternal coronavirus infections and pregnancy outcomes. Arch Pathol Lab Med. 2020 Mar 17. doi: 10.5858/arpa.2020-0901-SA.

37. Diagnóstico SARS-CoV-2 para embarazadas en situación de parto. Documento conjunto SOCHOG - SOCHINF - SCAI mayo 22, 2020 https://sochog.cl/wpcontent/uploads/2020/05/Concenso-2.0.pdf 


\section{FIGURAS Y TABLAS}

Figura 1. Mapa de las instituciones participantes en la presente publicación $(n=23)$.

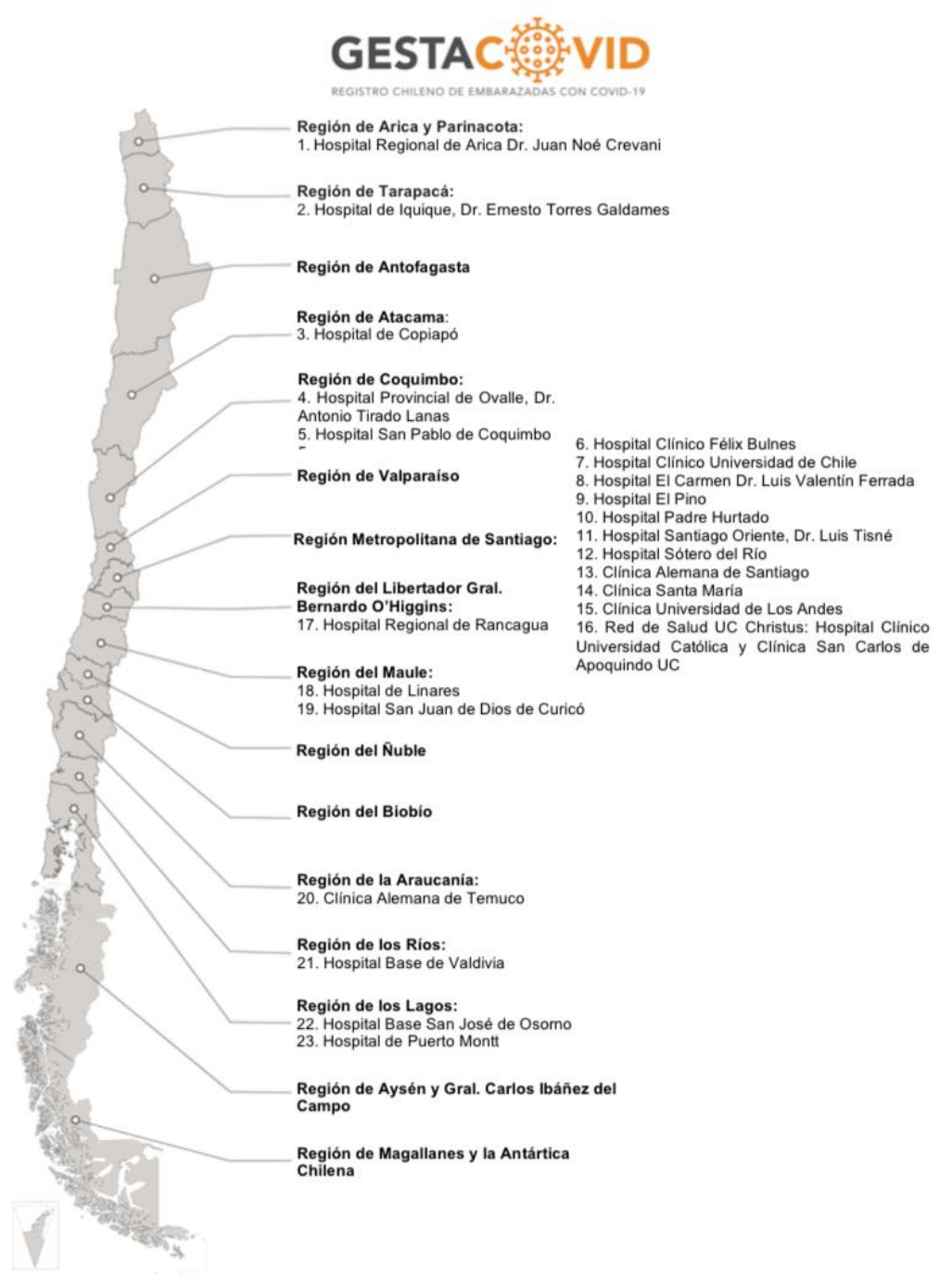


Figura 2. Gráfico de representación de síntomas principales mediante un diagrama de efectos o forest plot, entre pacientes hospitalizadas y ambulatorias).

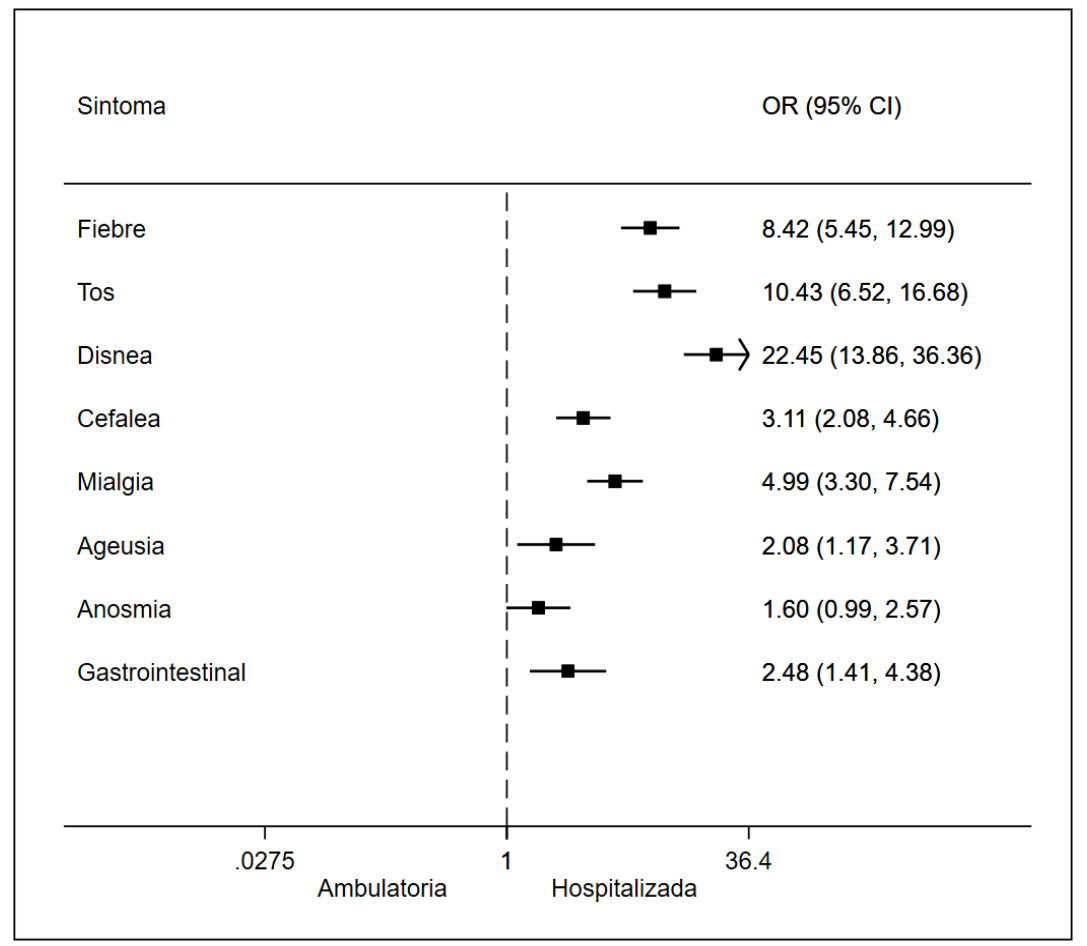

Tabla 1. Distribución de las embarazadas con COVID-19 según institución y motivo de estudio.

\begin{tabular}{lcccc}
\hline \multicolumn{1}{c}{ Institución } & $\begin{array}{c}\text { Cribado } \\
\text { universal } \\
\%(\mathbf{n})\end{array}$ & $\begin{array}{c}\text { Contacto } \\
\text { directo } \\
\%(\mathbf{n})\end{array}$ & $\begin{array}{c}\text { Sintomáticas } \\
\%(\mathbf{n})\end{array}$ & $\begin{array}{c}\text { Total global } \\
\%(\mathbf{n})\end{array}$ \\
\hline ZONA NORTE & $14 \%(1)$ & $43 \%(3)$ & $43 \%(3)$ & $1 \%(7)$ \\
Hospital de Arica & $15 \%(6)$ & $28 \%(11)$ & $57 \%(23)$ & $6 \%(40)$ \\
Hospital de Iquique & $100 \%(3)$ & 0 & 0 & $0,4 \%(3)$ \\
Hospital de Copiapó & $100 \%(3)$ & 0 & 0 & $0,4 \%(3)$ \\
Hospital de Ovalle & $37,5 \%(3)$ & $37,5 \%(3)$ & $25 \%(2)$ & $1 \%(8)$ \\
Hospital de Coquimbo & & & & $6 \%(41)$ \\
ZONA CENTRO & $66 \%(27)$ & $2 \%(1)$ & $32 \%(13)$ & $6 \%(53)$ \\
Hospital Clínico U. de Chile & $21 \%(11)$ & $4 \%(2)$ & $75 \%(40)$ & $8 \%(3)$ \\
Hospital El Carmen & $52 \%(36)$ & $4 \%(3)$ & $44 \%(30)$ & $10 \%(69)$ \\
Hospital El Pino & $76 \%(96)$ & $5 \%(6)$ & $19 \%(24)$ & $19 \%(126)$ \\
Hospital Félix Bulnes & $13 \%(2)$ & $6 \%(1)$ & $81 \%(13)$ & $2 \%(16)$ \\
Hospital Luis Tisné & & & & \\
\hline
\end{tabular}




\begin{tabular}{lcccc} 
Hospital Padre Hurtado & $28 \%(21)$ & $12 \%(9)$ & $60 \%(45)$ & $11 \%(75)$ \\
Hospital Sótero del Río & $3 \%(2)$ & $9 \%(6)$ & $88 \%(57)$ & $10 \%(65)$ \\
Red de Salud UC-Christus & $39 \%(15)$ & $8 \%(3)$ & $53 \%(20)$ & $6 \%(38)$ \\
Clínica Alemana de & $4 \%(2)$ & $20 \%(9)$ & $76 \%(34)$ & $7 \%(45)$ \\
Santiago & $81 \%(13)$ & $13 \%(2)$ & $6 \%(1)$ & $2 \%(16)$ \\
Clínica Santa María & $29 \%(2)$ & $14 \%(1)$ & $57 \%(4)$ & $1 \%(7)$ \\
Clínica U. de Los Andes & $25 \%(2)$ & 0 & $75 \%(6)$ & $2 \%(8)$ \\
Hospital de Rancagua & $17 \%(2)$ & $25 \%(3)$ & $58 \%(7)$ & $2 \%(12)$ \\
Hospital de Curicó & 0 & $100 \%(1)$ & 0 & $100 \%(1)$ \\
Hospital de Linares & & $14 \%(1)$ & $14 \%(1)$ & $1 \%(7)$ \\
ZONA SUR & $72 \%(5)$ & $57 \%(4)$ & $1 \%(7)$ \\
Clínica Alemana de Temuco & 0 & 0 & $100 \%(7)$ & $1 \%(7)$ \\
Hospital Base de Valdivia & 0 & $14 \%(1)$ & $14 \%(1)$ & $1 \%(7)$ \\
\hline Hospital de Osorno & $72 \%(5)$ & $\mathbf{1 0 \% ( 6 9 )}$ & $\mathbf{5 1 \% ( 3 3 5 )}$ & $\mathbf{1 0 0 \% ( 6 6 1 )}$ \\
Hospital de Puerto Montt & $\mathbf{3 9 \% ( 2 5 7 )}$ & & & \\
\hline Total Global & & & & \\
\hline
\end{tabular}

Tabla 2. Características demográficas, clínicas y del diagnóstico de COVID-19 de la población en estudio $(n=661)$.

\begin{tabular}{lc}
\hline Característica & Mediana (RI) - \% (n/N) \\
\hline Edad materna (años) & $29,9(25,2-34,4)$ \\
Patología crónica: & \\
DM tipo 1 y 2 & $3 \%(22 / 660)$ \\
HTA crónica & $5 \%(31 / 660)$ \\
IMC (kg/m2) & $30,4(26,7-34,5)$ \\
Estado nutricional al diagnóstico: & \\
Bajo peso & $0,2 \%(1 / 598)$ \\
Normal & $13,8 \%(83 / 598)$ \\
$\quad$ Sobrepeso & $33 \%(200 / 598)$ \\
Obesidad & $53 \%(314 / 598)$ \\
EG al diagnóstico (semanas) & $34,6(26,6-38,3)$ \\
Diagnóstico: & \\
1er trimestre & $8 \%(52 / 661)$ \\
2do trimestre & $18 \%(119 / 661)$ \\
3er trimestre & $68 \%(450 / 661)$ \\
Puerperio & $6 \%(40 / 661)$
\end{tabular}


Forma de diagnóstico:

$\begin{array}{cc}\text { PCR hisopado } & 99 \%(655 / 661) \\ \text { TAC/Rx de tórax compatible } & 0,8 \%(5 / 661) \\ \text { Serología } & 0,2 \%(1 / 661) \\ \text { Hospitalización por COVID: } & 19 \%(123 / 659) \\ \text { Sala básica } & 61 \%(75 / 123) \\ \text { UPC } & 39 \%(48 / 123) \\ \text { Embarazo en curso } & 38 \%(251 / 661)\end{array}$

EG: edad gestacional; DM: diabetes mellitus; HTA: hipertensión arterial. Variables continuas expresadas en mediana (rango intercuartil); variables categóricas expresadas en \% $(n / N)$.

Tabla 3. Frecuencia de los signos y síntomas de pacientes embarazadas con infección por SARS-CoV-2, en forma global y según manejo de la patología.

\begin{tabular}{|c|c|c|c|c|}
\hline $\begin{array}{l}\text { Síntomas y } \\
\text { Signos }\end{array}$ & $\begin{array}{c}\text { Global } \\
(n=659)^{\dagger}\end{array}$ & $\begin{array}{c}\text { Manejo } \\
\text { ambulatorio } \\
(n=536)\end{array}$ & $\begin{array}{c}\text { Manejo } \\
\text { hospitalizada } \\
(n=123)\end{array}$ & Valor $\mathbf{p}^{*}$ \\
\hline Tos & $35 \%$ (232) & $25 \%(136)$ & $78 \%(96)$ & $<0,0001$ \\
\hline Mialgia & $31 \%(207)$ & $24 \%(131)$ & $62 \%(76)$ & $<0,0001$ \\
\hline Cefalea & $30 \%(202)$ & $26 \%(138)$ & $52 \%(64)$ & $<0,0001$ \\
\hline Fiebre & $29 \%(193)$ & $20 \%(109)$ & $68 \%(84)$ & $<0,0001$ \\
\hline Disnea & $21 \%(139)$ & $10 \%(52)$ & $71 \%(87)$ & $<0,0001$ \\
\hline Anosmia & $18 \%(117)$ & $16 \%(88)$ & $24 \%(29)$ & 0,052 \\
\hline Ageusia & $10 \%(63)$ & $8 \%(44)$ & $16 \%(19)$ & 0,01 \\
\hline Síntomas Gl & $9 \%(62)$ & $7 \%(41)$ & $17 \%(21)$ & 0,001 \\
\hline
\end{tabular}

GI: gastrointestinal. Datos expresados en $n^{\circ} \%$ (n). †La información de los síntomas no se encuentra disponible en dos casos. *Comparación de proporciones en pacientes con manejo ambulatorio versus hospitalizadas (test chi2). 
Tabla 4. Resultados maternos y perinatales de las pacientes que presentaron infección por SARS-CoV-2 durante el embarazo.

\begin{tabular}{|c|c|}
\hline Resultados maternos y perinatales & \% (n) - mediana (RIQ). \\
\hline \multicolumn{2}{|l|}{ Vía del parto $(n=391)$ : } \\
\hline Vaginal espontáneo & $42.8 \%(174)$ \\
\hline Instrumental & $1 \%(4)$ \\
\hline Cesárea & $51,4 \%(209)$ \\
\hline Sin información & $1 \%(4)$ \\
\hline Abortos & $4 \%(16)$ \\
\hline Edad gestacional al parto (semanas) & $38.6(37.3-39.6)$ \\
\hline Parto prematuro (< 37 semanas) & $17 \%(68)$ \\
\hline Interrupción por COVID-19 & $8 \%(33)$ \\
\hline Morbilidad materna grave & $12 \%(49)$ \\
\hline Peso del RN (gramos) & $3270(2830-3600)$ \\
\hline RN PEG & $9 \%(38)$ \\
\hline PCR (+) recién nacido & $5 \%(21)$ \\
\hline Muerte perinatal & $1 \%(6)$ \\
\hline
\end{tabular}

RN: recién nacido; PEG: pequeño para la edad gestacional. Datos expresados en \% ( $n$ ) o mediana (rango intercuartil).

Tabla 5. Características de los partos de mujeres con COVID-19 cuyos recién nacidos tuvieron una PCR positiva para SARS-CoV-2 durante las primeras 72 horas del parto.

\begin{tabular}{ccccccc}
\hline $\begin{array}{c}\mathbf{N}^{\circ} \\
\text { caso }\end{array}$ & $\begin{array}{c}\text { EG al } \\
\text { diagnóstico }\end{array}$ & $\begin{array}{c}\text { Indicación del } \\
\text { estudio }\end{array}$ & $\begin{array}{c}\text { Momento de toma } \\
\text { PCR desde el parto } \\
\text { (hrs) }\end{array}$ & $\begin{array}{c}\text { EG al } \\
\text { parto }\end{array}$ & $\begin{array}{c}\text { Vía del } \\
\text { parto }\end{array}$ & $\begin{array}{c}\text { Peso del } \\
\text { RN }\end{array}$ \\
\hline 1 & $33+6$ & Cribado & $0-12$ & $34+1$ & Vaginal & 2435 \\
2 & $39+4$ & Cribado & $>48$ & $39+5$ & Cesárea & 3870 \\
3 & $38+1$ & Cribado & $0-12$ & $38+1$ & Cesárea & 3066 \\
4 & Puérpera & Sintomática & s/d & $38+1$ & Cesárea & 3730 \\
5 & $38+5$ & Cribado & $0-12$ & $38+5$ & Cesárea & 3428 \\
6 & $38+5$ & Cribado & $0-12$ & $39+1$ & Cesárea & 3500 \\
7 & $39+4$ & Cribado & $0-12$ & $40+0$ & Vaginal & 3282 \\
8 & $38+1$ & Cribado & $12-24$ & $38+1$ & Cesárea & 4345 \\
9 & $39+1$ & Cribado & $12-24$ & $39+1$ & Vaginal & 3715 \\
10 & Puérpera & Contacto & $0-12$ & $38+4$ & Cesárea & 3500 \\
11 & $37+6$ & Cribado & s/d & $37+6$ & Cesárea & 3100 \\
\hline
\end{tabular}




\begin{tabular}{lcccccc}
12 & $40+3$ & Cribado & $0-12$ & $40+4$ & Cesárea & 4115 \\
13 & $40+0$ & Cribado & $>48$ & $40+0$ & Vaginal & 3745 \\
14 & Puérpera & Cribado & $0-12$ & $38+5$ & Vaginal & 3810 \\
15 & $40+0$ & Cribado & $>48$ & $40+0$ & Cesárea & 3800 \\
16 & Puérpera & Cribado & $>48$ & $38+5$ & Vaginal & 2590 \\
17 & $41+0$ & Cribado & $0-12$ & $41+0$ & Cesárea & 3934 \\
18 & $35+6$ & Cribado & s/d & $35+6$ & Cesárea & 2504 \\
19 & $39+2$ & Sintomática & s/d & $39+3$ & Cesárea & 3345 \\
20 & $38+1$ & Sintomática & $>48$ & $38+2$ & Cesárea & 3475 \\
21 & $32+0$ & Cribado & $0-12$ & $35+1$ & Cesárea & 2650 \\
\hline
\end{tabular}

EG: edad gestacional; RN: recién nacido.

Anexo I. Colaboradores Grupo GESTACOVID.

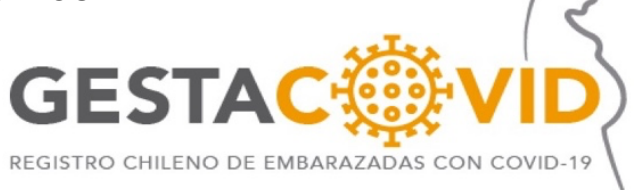

Anthara Soto Z. (Hospital de Copiapó)

Carlos Trujillo N. (Hospital Félix Bulnes)

Carolina San Martín J. (Hospital Gustavo Fricke)

Consuelo Sobarzo U. (Hospital El Carmen de Maipú.)

Daniela Burky A. (Hospital Dr. Luis Tisné Brousse)

Danisa González V. (Hospital Regional de Iquique Dr. Ernesto Torres Galdames)

Diane Gutiérrez C. (Hospital Clínico Universidad de Chile)

Dr. Ernesto Torres Galdames)

Francisca González T. (Hospital El Pino)

Héctor Eliash D. (Hospital El Pino)

Horacio Figueroa D. (Clínica Universidad de los Andes)

Iván Rojas T. (Clínica Santa María.)

Jean Pierre Frez B.(Hospital de Puerto Montt, Dr. Eduardo Schütz Schroeder)

Jorge Carrillo O. (Hospital Base San José de Osorno)

José Andrés Poblete L. (Pontificia Universidad Católica de Chile)
José Martínez M. (Hospital de Linares Carlos Ibáñez del Campo)

Juan Muñoz R. (Hospital Regional de Temuco)

Julio Astudillo D. (Clínica Alemana de Santiago)

Karen Rehren L. (Hospital Regional de Temuco)

Karla Silva (Hospital Dr. Sótero del Río)

Margot Acuña S.(Hospital Regional de Temuco)

María José Aguilera C.(Hospital Félix Bulnes)

Marta Sánchez M. (Clínica Alemana de Santiago)

Martín Del Río D. (Hospital Regional de Talca)

Mónica Acevedo D. (Hospital Dr. Luis Tisné Brousse)

Rocío Naveas P. (Pontificia Universidad Católica de Chile)

Rodrigo Latorre R. (Hospital Padre Hurtado)

Trinidad Astete W. (Hospital de Puerto Aysén)

Víctor Córdova P. (Hospital Dr. Sótero del Río) 